\title{
Hybrid nanofluid flow and heat transfer over backward and forward steps: a review
}

\begin{abstract}
This work describes the recent progress in the improvement of heat transfer through microscale facing steps. This analysis includes previous studies that aimed to improve heat transfer with and without hybrid nanofluids. This review presents the experimental and numerical results on the usage of hybrid nanofluids. Furthermore, this work introduces the use of backward-facing step (BFS), forward-facing step (FFS) and microscale steps with different flow regimes and working fluids. This study reveals an increase in heat transfer by utilizing hybrid nanofluids as a working fluid and an improvement in the coefficient of heat transfer when the nanoparticle volumes and concentrations of hybrid nanofluids increase. This work points out the studies on hybrid nanofluids over BFS and FFS, describes various nanoparticles used on the basis of thermal conductivity and shows the improvement in the rate of heat transfer. This study also outlines the discussion and future direction of the current review.
\end{abstract}

Keyword: Heat transfer enhancement; Backward-facing steps; Forward-facing steps; Hybrid nanofluids 